\title{
Las relaciones entre México y Estados Unidos en la era de la globalización
}

\author{
Steven C. Topik \\ DEPARTAMENTO DE HISTORIA \\ UNIVERSIDAD DE CALIFORNIA, IRVINE
}

La "globalización" presenta grandes retos a los estudiosos de las relaciones México-Estados Unidos al cuestionar el papel del Estado, las ideas de nación y región, y reconsiderar las fuerzas causales, las categorías y la medida del análisis. Mucho de esto no es nuevo y sin precedentes. El pasado es una buena guía para entender el presente, y éste nos ayuda a comprender el pasado. Estudiar las relaciones internacionales debería ser vital para comprender la interrelación entre dónde estamos y a dónde vamos.

$\mathrm{L}$ a moda actual de hablar de la "globalización" representa retos importantes para aquellos que estudian las relaciones entre México y Estados Unidos. Ella cuestiona el papel del Estado, las ideas de nación y región, y exige reconsiderar las fuerzas causales, las categorías y la medida del análisis. Los eruditos están guiados por el nuevo discurso que exalta los "mercados libres" y un gobierno limitado. A esto se le llama "globalización", una fuerza seductora que ha sido desencadenada por las "leyes del mercado" y por empresarios privados más que por los Estados. Con mucha frecuencia, los comentaristas anuncian que esta configuración constituye un mundo nuevo, diferente del pasado. ${ }^{1}$ No han buscado suficientemente lejos. Es cierto que existen precedentes.

Voy a sugerir que la actual globalización no es algo nuevo y sin precedentes. De hecho, el pasado es una guía para entender el presente. Y el presen-

\footnotetext{
${ }^{1}$ Traducción de Sergio Fernández Bravo. Véase, por ejemplo, Friedman, Lexus, 1998, y Thurow, Building, 1999, así como la penetrante revisión sobre ambos, en Frank, "Globalicious", 1999, pp. $72-75$.
} 
te nos ayuda a buscar en los sitios precisos para entender el pasado. Algunos de los acontecimientos actuales que están transformando nuestra manera de considerar categorías como fronteras, etnicidad, nacionalidad, fronteras públicas y privadas y organizaciones no gubernamentales, y la importancia de los intercambios culturales, deben proyectarse hacia atrás en el tiempo.

Analizaré en especial los estudios de las relaciones México-estadunidenses hechos en Estados Unidos, pues es ahí donde radica mi ventaja comparativa; no tendría demasiado sentido para mí escribir sobre lo que se está haciendo en México.

Por tradición, los estudios de las relaciones exteriores de Estados Unidos se han concentrado en el expansionismo, ya sea territorial o neocolonial. Una debilidad seria en el debate sobre el expansionismo estadunidense ha sido la tendencia a enmarcarlo por completo en términos de sus preocupaciones e intereses. Las otras naciones son tratadas principalmente como bolas de billar, sólo interesantes cuando chocan con Estados Unidos.

Algunas de ellas, como las grandes potencias europeas, aparecen como las bolas clave que inician el movimiento; otras, como las naciones latinoamericanas, sólo rebotan cuando se les golpea. Se hace poco esfuerzo por mirar dentro de las bolas para entender qué es lo que las mueve. La escasa literatura de América Latina sobre la historia de las relaciones internacionales tiende también a ver a Estados Unidos como el agresor y a los latinoamericanos como las víctimas; víctimas tanto de la fuerza estadunidense como de las eli- tes de compradores domésticos. ${ }^{2}$ De hecho, a menudo se involucran fuerzas mucho más complejas y percepciones más ilustradas.

El enfoque de la bola de billar revela una tendencia todavía popular de privilegiar los Estados-nación y considerarlos como monolíticos y gobernados desde la capital por un pequeño núcleo. Después de todo, la historia diplomática nació en el siglo XIX, cuando la construcción del Estado y el nacionalismo eran los temas dominantes. De ahí que eruditos y periodistas escriban sólo sobre México o sobre Estados Unidos. Ello se reduce a veces a México contra Washington o, en la actualidad, a Zedillo contra Clinton. Aunque se puede entender que los periodistas, con sus fechas de entrega apretadas y sus limitaciones de espacio utilicen esta taquigrafía, tiene mucho menos disculpa que lo hagan los eruditos. Las naciones no son una entidad monolítica y con un propósito único, sino más bien un atado de intereses a menudo contradictorios e inestables. $Y$ es cierto que no todas las interacciones internacionales están necesariamente en el nivel de las naciones.

\section{EL EXPANSIONISMO DE ESTADOS UNIDOS}

El tema guía de los asuntos exteriores de Estados Unidos es el expansionismo. Esto es razonable, ya que Estados Unidos ha sido largo tiempo una nación expansionista. Aun cuando empezó su expansión y su movimiento ha-

\footnotetext{
${ }^{2}$ Entre las obras que adoptan esta perspectiva está Bandeira, Presença, 1967.
} 


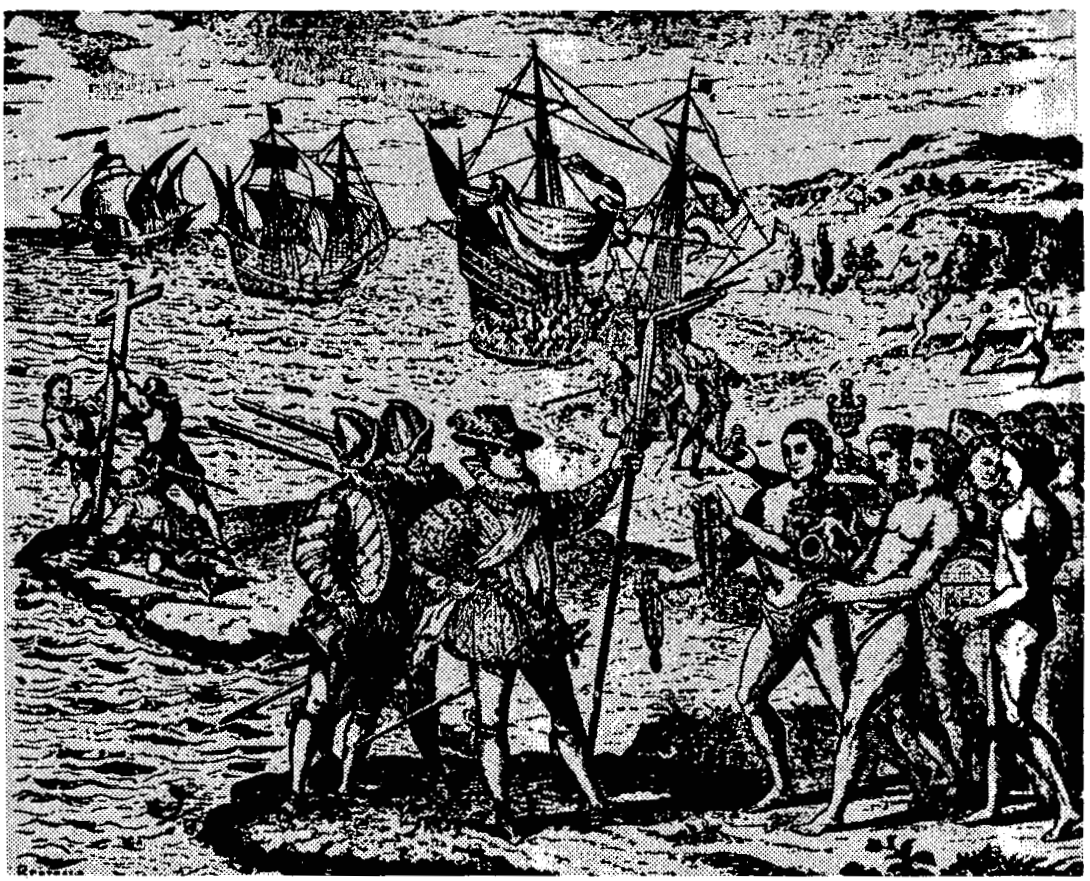

cia las tierras reclamadas por España mucho antes de convertirse en una nación independiente, esta expansión fue concebida como "continentalismo" hasta los años 1890. A partir de la llegada de los colonialistas a Jamestown, los súbditos británicos se diseminaron con lentitud a lo largo de la costa y hacia el interior del vasto continente estadunidense. Pero tras 200 años de gobierno británico aproximadamente, las colonias tenían cerca de 5000000 de habitantes, en su mayoría apiñados en el Atlántico con unas cuantas avanzadas en el interior. Entonces, en las primeras seis décadas del siglo XIX, los jóvenes Estados Unidos adquirieron y se apoderaron de territorio a una de las mayores velocidades en la historia. Las compras a España, Francia y Rusia, y las apropiaciones a costa de México, extendieron la autoridad de Washington de jure, si no de facto, del Atlántico al Pacífico. Los asuntos exteriores se interesaban principalmente en proteger los litorales de las potencias europeas y en la dirección de sangrientas guerras contra los habitantes indígenas de esas tierras que con tanta facilidad fueron cedidas por distantes poderes 
europeos. Estados Unidos engrosaba con la mayor inmigración transoceánica de la historia, que llevó cerca de 25000000 de europeos. Al mismo tiempo, la población euroamericana gozaba de una de las mayores tasas de crecimiento natural del mundo. La población se duplicó cada 23 años. Oleadas de individuos se movian hacia las tierras de los nativos americanos, empujando la frontera europeizada siempre hacia el oeste.

Con las fronteras del país protegidas de las grandes potencias, los 35 años anteriores a la guerra civil fueron un tiempo de consolidación e integración económica. Entre 1865 y 1900 se había labrado tanta tierra como la cultivada en los dos y medio siglos anteriores. Se dedicaba el triple de tierra al cultivo del algodón en $\mathbf{1 8 9 0}$ de aquella cultivada durante la cúspide del periodo antibélico. El maíz, el trigo y el tabaco fueron más que duplicados. Durante el mismo periodo las manufacturas se extendieron más de cuatro veces, causando que las ganancias agrícolas del producto interno bruto cayeran de un tercio en 1869 a menos de un cuarto en 1890. El mercado interno se convirtió en el mayor del mundo cuando los fabricantes y los granjeros comerciales quedaron unidos por la mayor red ferroviaria, la cual se cuadruplicó entre 1870 y 1890 .

Esto permitió que el centro de gravedad demográfico del país se moviera hacia el oeste. Los trece estados originales, que en 1800 contenían $93 \%$ de la población europeizada, reunían sólo $50 \%, 60$ años después, y $41 \%$ en 1890 . El medio oeste casi igualaba en habitantes a la costa este en ese último año.
La expansión hacia el oeste, dentro de la psique nacional, significaba más que un movimiento demográfico, era una misión nacional. Los ciudadanos de Estados Unidos consideraban que su embestida no equivalía a una guerra de conquista, sino a una misión divinamente ordenada para poblar el continente "vacío" y domeñar la frontera. Anticipándose a Herbert Spencer, muchos estadunidenses consideraban la conquista continental como evidencia de su rectitud y potencia y de ser un pueblo escogido por Dios. Como lo proclamaba el presidente James $\mathrm{K}$. Polk, la ocupación de tierras aledañas era el Destino Manifiesto de Estados Unidos. Este continentalismo fue considerado por lo general como la construcción de una nación, no como la construcción de un imperio. (A diferencia de los rusos, que al mismo tiempo avanzaban sobre los pueblos de Asia central y los integraban como unidades de su imperio, Estados Unidos marginaba, borraba o exterminaba a los numerosos y menos complejos pueblos indígenas.) Los estadunidenses inventaron el mito del "crisol de razas" para acentuar la homogeneidad, aun cuando su política hacia los nativos americanos se acercaba más a la "limpieza étnica". A causa de que la conquista del oeste fue realizada en su mayor parte por colonizadores privados, más que por medio de campañas militares, la imagen prevaleciente de.la frontera fue la heredad fuerte y autosuficiente del pionero más que la delcampo de batalla y el cuartel militar. ${ }^{3}$ La marcha ha-

${ }^{3}$ Jones, Presidential, 1964, pp. 351-389. 
cia el Pacífico creó, no una burocracia imperial con legiones de soldados y recolectores de impuestos como ocurrió con la creación de naciones en Europa, sino más bien produjo un gobierno descentralizado y localizado, al que Ann Markusen menciona como el "Estado territorializado". ${ }^{4}$ Los estadunidenses desconfiaban del ya alejado gobierno nacional. El peculiar estilo de frontera en la construcción de Estados Unidos como nación hizo que la conquista de los pueblos vecinos se viera como un proceso civil orgánico interno, no como un asunto extranjero estatal. $Y$ a pesar de tener una herencia de conquista y expansión, los estadunidenses veían con cautela las colonias de ultramar y un Estado imperial.

En consecuencia, antes de los años 1880 el país se miraba a sí mismo como aislacionista en relaciones exteriores, aun cuando de hecho estaba íntimamente involucrado con el comercio internacional, las inversiones y la inmigración. Las interacciones con extranjeros solían ser vistas como parte de un proceso natural en la construcción del Estado y la nación. Sus acciones estaban relacionadas en su mayor parte con la protección y la expansión de su proyecto continental. Sin embargo, la toma de California tras la derrota de México en 1848, redefinió los intereses continentales. El descubrimiento del oro en 1848 transformó la soñolienta y escasamente poblada costa del Pacífico en un imán capaz de atraer a otros individuos. Al cabo de un año, luego del hallazgo de oro por James W. Marshall,

\footnotetext{
${ }^{4}$ Markusen, Regions, 1987, p. 33.
}

la población no nativa de California aumentó de 14000 a 100000 . Casi se cuadruplicó en la siguiente década. Trabajadores chinos, trigo chileno y maíz salvadoreño llegaron para satisfacer la demanda creciente.

La riqueza recién hallada inclinó un poco hacia el oeste a Estados Unidos. Ahora los intereses nacionales incluían la defensa de la costa occidental; más aún, con el país convertido en una potencia del Pacífico, los países asiáticos eran tan vecinos como los europeos. La forzada apertura de Japón por el almirante Perry en 1854, pisándole los talones a la apertura inglesa del mercado de China por medio de las guerras del Opio, mostró la nueva preocupación y nueva imagen de sí mismo de Estados Unidos. Quizá con mayor importancia, su expansión transcontinental necesitaba medios para conectar las dos costas. Antes de que el ferrocarril uniera este y oeste en Promontory Point, Utah, en 1869, las compañías estadunidenses y británicas competían por rutas de canal y de ferrocarril a través de México, Nicaragua y Panamá. De esta manera, América Central adquirió un interés estratégico. Y en forma similar. las islas del Caribe ganaron importancia como lugares potenciales de bases navales para la defensa del proyectado canal. No obstante, la política caribeña y la centroamericana se concebían por lo general como extensiones de la política nacional, más que como asuntos exteriores cualitativamente diferentes. ${ }^{5}$

\footnotetext{
${ }^{5}$ Véanse los artículos de Ana Rosa Suárez, "Peter A. Hargous", y Samantha Álvarez, "El paso interoceánico", publicados en este mismo número.
} 
De manera lenta, la necesidad de una expansión hacia el exterior surgió del fortalecimimiento de la ocupación del continente por Estados Unidos y de problemas económicos en su interior. El periodo entre 1873 y 1896 ha sido llamado de la "Gran Depresión". Aun cuando el producto nacional bruto per capita real creció cerca de $50 \%$ durante el periodo, la era fue marcada por la mayor depresión conocida por el país entre 1873 y 1877 , una contracción entre 1887 y 1891 , a la que siguió una baja más severa entre 1893 y 1895.

Hacia los años 1890, un creciente número de estadunidenses refórmuló la misión de Estados Unidos y su papel en el mundo. Este periodo manifestó asuntos similares a los de hoy, envueltos en un lenguaje diferente, que pre. ocuparon a políticos y hombres de negocios. Estados Unidos se proyectó primero en la escena internacional, esperando demarcar una esfera de influencia en América Latina. ${ }^{6}$ No contento ya con ser un experimento del nuevo mundo como una "ciudad en una colina", ni satisfecho con que el destino manifiesto de la nación fuera extenderse y desarrollar el continente del Atlántico al Pacífico, empezó a mirar hacia el exterior. Cada vez más estadunidenses fueron seducidos por los mercados extranjeros, los territorios, las bases navales y los posibles conversos religiosos.

La extensión del sentimiento expansionista en Estados Unidos y los orígenes del mismo han sido muy debatidos. No se discute que en los alegres años noventa el país ganara cerca de 100000 millas cuadradas en territorios de ultra-

\footnotetext{
'Topik, Trade, 1996.
}

mar al apoderarse de Cuba, Guam, Hawai, las Filipinas, Puerto Rico y las Islas Vírgenes como colonias o protectorados, y que se preparase para ser el "policía del Caribe". Pero hay desacuerdo sobre cuáles de las adquisiciones territoriales fueron un accidente histórico o un componente inevitable del desarrollo capitalista y un resultado integral de la estructura de la sociedad y el pensamiento de Estados Unidos. La combinación de "expansionismo" y "anexión" ha impedido a los historiadores estudiar detalladamente las iniciativas orientadas hacia el mercado que no tienen aspiraciones territoriales. ${ }^{7}$

Los defensores del punto de vista accidental sostienen que las relaciones extranjeras no interesaron mayormente a políticos, hombres de negocios o al público de Estados Unidos. El mercado nacional y la política interior atrapaban su atención. A diferencia de la Europa imperialista, los asuntos extranjeros eran de importancia limitada, marginal y esporádica en los Estados Unidos republicanos. ${ }^{8}$ Sólo después de que los

\footnotetext{
7 Véase el debate entre Field, "American", 1978, pp. 669-674, y Beisner, "Comment", 1978, pp. 674-679. Benjamin, "Framework", 1987, pp. 91, 95, da al traste con el argumento accidental cuando señala que, de hecho, en el siglo xIX Estados Unidos era "la nación más consistentemente expansionista de los tiempos modernos" porque más que negociar con los ciudadanos de los países adyacentes, simplemente los conquistaba. Sólo a fines del siglo XIX ya no se consideró necesaria o justificable la ocupación física. En vez de ella se acudió al comercio, la inversión extranjera y los misioneros.

${ }^{8}$ Bemis, Diplomatic, 1936; Holbo, "Economics", 1971, pp. 199-221; Graebner, Uncertain, 1961. Señalado también en Gardner, Creation, 1973.
} 
territorios de ultramar cayeron en su inmenso regazo, los ciudadanos del nuevo imperio lucharon por definir el nuevo papel internacional del país.

Otros historiadores, como William Appleman Williams, Walter LaFeber y Thomas J. McCormick argumentan, por lo contrario, que el siglo XIX fue testigo de una transformación fundamental de la economía y la sociedad de Estados Unidos que hizo necesario el expansionismo hacia el exterior. ${ }^{9}$ Parte de su argumento sigue la noción de Charles Beard sobre el desarrollo del interés nacional y también se apoya en el excepcionalismo. La frontera de Estados Unidos, que se supone hizo tanto para forjar el carácter de la nación, fue percibida en forma popular como terminada en los años 1890. Esto generó temor de que la "válvula de seguridad" democrática estuviera apagada; la inquietud popular, ya evidente en el surgimiento de los Caballeros del Trabajo, y los movimientos de La Granja y Populista pudieron hacer explosión sin la alternativa fronteriza. El celo misionero que predicaba que el Destino Manifiesto de la nación era llenar el continente, creía entonces que los hombres blancos del país debían aceptar la carga de mejorar a los pueblos no civilizados de otros países, de manera que Estados Unidos pudiese "diseminar el sueño americano". ${ }^{10}$ De esta manera, la cultura se vuelve un importante instrumento explicativo para estos historiadores diplomáticos.

\footnotetext{
"Williams, Tragedy, 1959; Lafeber, New, 1963, y "Comment", vól. 2, 1993; McCormick, Cbina, 1967.

${ }^{10}$ Rosenberg, Spreading, 1982.
}

Otra parte del argumento expansionista de Williams, LaFeber y McCormick sostiene que la política exterior de Estados Unidos estuvo sujeta a las mismas fuerzas económicas que condujeron al imperialismo europeo. Sostienen, como lo hicieron J. A. Hobson y Rosa Luxemburgo en sus estudios sobre el imperialismo europeo, que el nuevo expansionismo era una manifestación de la necesidad de los países capitalistas desarrollados de encontrar nuevos mercados. Los nuevos mercados se volvieron necesarios porque, a medida que el capitalismo alcanzaba su estado monopólico, concentró la riqueza en las manos de los capitalistas y privó a los trabajadores del suficiente poder adquisitivo. Como lo demostró la destructiva depresión de los años 1870 , los mercados se saturaron y los precios se desplomaron. Además de los problemas causados por mercados llenos de mercancías, había un sobreflujo de capital que hizo bajar las tasas de interés. La avanzada industrialización también requirió de mayores y más confiables materias primas y los precios declinantes necesitaban insumos más baratos. Luxemburgo, Hobson y Lenin consideraron que, por tanto, el capitalismo avanzado condujo a las potencias europeas al colonialismo.

Más afines con la experiencia de Estados Unidos son los estudios de Ronald Robinson y John Gallegher quienes, al estudiar el imperio británico en África, mantienen que la reivindicación de una presión política para obtener ventajas económicas no requirió necesariamente de colonias formales. En realidad, el "imperialismo del mercado libre", que alcanzó los mercados sin un control político directo, era preferible 
en muchos aspectos al gobierno directo. ${ }^{11}$ Muchos artífices políticos de finales del siglo XIX en Estados Unidos compartieron esta opinión. Ellos buscaron aliados extranjeros que sirvieron como colaboradores tanto para promover sus propios intereses como los de Estados Unidos. Las escuelas antiimperialista y de la dependencia subrayaron las estructuras de la economía internacional y de la economía política, considerando que el mercado mundial impulsaba a la diplomacia.

Mis propias investigaciones sobre Estados Unidos y Brasil en el periodo 1888-1896 sugieren que es mejor un punto intermedio entre las escuelas estructurales y de la contingencia, entre las explicaciones económicas y las políticas. ${ }^{12}$ Los debates sobre las relaciones exteriores de Estados Unidos se han volcado sobre esos asuntos como la importancia relativa de las consideraciones económicas, políticas y estratégicas, así como el peso relativo de las estructuras sociales o la influencia de individuos específicos cuando se decide la política exterior. Por lo general, los historiadores que subrayan las motivaciones económicas en la política exterior utilizan el análisis de clase y señalan las necesidades estructurales de una economía en expansión. Los historiadores de la política, por otra parte, son mucho más parciales sobre las maquinaciones de algunos individuos para explicar los resultados. Los historiadores militares se interesan por las exigencias tecnológicas pero también resaltan el

\footnotetext{
${ }^{11}$ Para un buen examen de esta postura véase Lewis, Imperialism, 1976.

12 Topik, Trade, 1996.
}

pensamiento y las acciones de individuos clave. Al llevarlas al campo internacional se perciben esas mismas tendencias. Acentuar las causas económicas es discutir las estructuras; resaltar las causalidades políticas es hablar de políticos individuales; los asuntos militares llaman la atención sobre el mate. rial de guerra y los estrategas.

Los hombres de negocios no están bien representados. En realidad, la visión general es que no se interesan en los asuntos exteriores hasta la conclusión de la Depresión de los años $1890{ }^{13}$ Aun aquellos que argumentan el significado del impulso expansionista en la cláusula de reciprocidad de la tarifa de McKinley sostienen que los políticos se adelantaban a la comunidad empresarial. Pero encuentro que, de hecho, Charles Flint, un exportador mayorista, importador, fletador, promotor de consorcios y tratante de armas, demostró que había un pequeño grupo de mercaderes con base en Nueva York y Boston que estaban muy interesados en los asuntos exteriores y eran muy efectivos en el cabildeo para lograr una política estatal expansionista. Estos hombres organizaron una campaña en la Conferencia Panamericana y en el Congreso de Estados Unidos para empujar a este país hacia el exterior. Y fueron todavía más activos en México que en Brasil.

Esto no significa que yo postule una teoría conspiratoria, sino más bien que amplío el concepto de liberalismo corporativo de Martin Sklar para que in-

${ }^{13}$ Campbell, Tranformation, 1976, p. 141; Holbo, "Economics", pp. 219-220; Becker, Dynamics, 1982, pp. XIV, 182; May, American, 1968 , p. 58. 


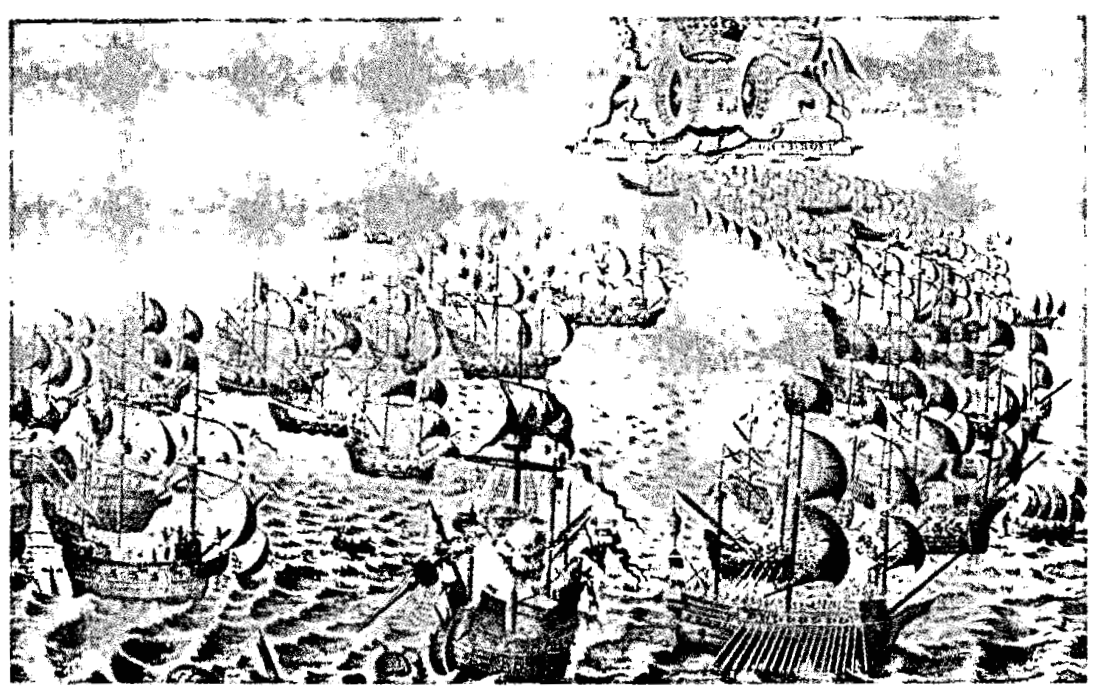

cluya la política exterior. En la Edad Dorada, los comités privados de "listón azul" y los consejos asesores tuvieron funciones quasi gubernamentales ya que los estadunidenses eran renuentes a fundar y habilitar una burocracia federal extensa. Estos hombres de negocios veían con naturalidad el interés nacional como su propio interés privado pero en mandato amplio. Su mezcla de actividades marciales, industriales y diplomáticas presagiaba el emergente complejo militar-industrial. ${ }^{14}$ Ello también señaló una importante vertiente en la transición, alejándose de los fili-

\footnotetext{
${ }^{14}$ Sklar, Corporate, 1988. Véase también Neison "Warfare", 1971, pp. 127.143, en donde se señala que el alboroto contra el matrimonio entre imperialismo y militarismo empezó en los años 1890.
}

busteros y aventureros económicos del siglo XIX que podian, como observa $\mathrm{V}$. K. Kiernan, "salirse con la suya sobornando como regla general a los jefes locales, tal como acostumbraban hacerlo en su casa". ${ }^{15}$ Ellos presagiaban a los "empresarios" del siglo XX, que utilizaban al gobierno de Estados Unidos para encontrar su camino en el exterior.

Lo mejor es tomar una posición intermedia entre el punto de vista del Estado como instrumental que se proyecta en el extranjero, y el del Estado céntrico. El Estado no era simplemente el comité central que defendía los intereses comunes de la burguesía, ya que los poseedores del capital estaban muy divididos entre sí en términos de intereses, percepciones de esos intereses, y

${ }^{15}$ Kiernan, Marxism, 1974 , p. 105 
nociones del papel correcto del Estado. Más aún, los administradores estatales, de alguna forma aislados de la sociedad civil por la confusión creada por los intereses contradictorios, tenían también sus propios intereses y puntos de vista sobre el interés nacional. Las políticas burocráticas eran importantes en el gobierno fragmentado y dividido de Estados Unidos. Sin embargo, la participación tras telones de los comerciantes de Nueva York y Boston hace que me detenga bruscamente ante la "visión intelectual que ve al Estado formulando en forma autónoma objetivos que luego intenta aplicar en contra de la resistencia de actores internacionales y locales" de Stephen Krasner. ${ }^{16}$ Tanto el Estado como la sociedad civil eran importantes en la formulación de la política exterior de Estados Unidos en México.

La materialización de Estados-nación ha llevado a una distorsión de la historia diplomática o de las relaciones internacionales, la que muy a menudo se enfoca sobre todo en el punto en donde las dos naciones se encuentran. Se supone que lo que está en juego son precisamente las negociaciones de los diplomáticos. Más productiva sería una economía política internacional que examinara los asuntos internos de cada país y sus efectos én la interrelación de ambos países. Un enfoque útil es lo que Stephen Gill ha llamado "his-

\footnotetext{
${ }^{16}$ Krasner, Defending, 1978, p. 11. Véase también Becker, Dynamics, quien argumenta, p. IX: "La burocracia gubernamental que nació para promover la expansión del comercio estadunidense no se créo simplemente como respuesta a las presiones de los negocios. La expansión económica estadunidense era parte de una lucha burocrática más amplia."
}

toricismo no estructural" gramsciano, que entiende el cambio histórico como el resultado de la actividad colectiva humana. ${ }^{17} \mathrm{Al}$ igual que la guerra es una extensión de la política, así también lo son las relaciones internacionales. Los regímenes internacionales modelan el discurso y las reglas del juego, pero las prioridades se determinan por intereses domésticos. ${ }^{18}$ Robert Putnam ha descrito apropiadamente las relaciones internacionales como un "juego en dos niveles":

A nivel nacional, los grupos domésticos persiguen sus intereses presionando al gobierno para que adopte politicas favorables, y los políticos buscan el poder construyendo coaliciones entre estos grupos. A nivel internacional, los gobiernos nacionales buscan maximizar su propia habilidad para satisfacer presiones internas, al tiempo que minimizan las consecuencias adversas de los desarrollos extranjeros. Ninguno de ambos juegos puede ser ignorado por los artífces centrales de las decisiones, en tanto que sus países siguen siendo interdependientes, y sin embargo soberanos. ${ }^{19}$

Putnam reconoce no sólo que los Estados tienen electorados competitivos, sino también que son fluidos. Sus apoyos y fines pueden cambiar el curso de las acciones emprendidas. No obstante, aunque esto sea con seguridad una mejoría sobre el enfoque de la bola de billar, ve aún lo nacional y al gobierno nacional como claramente monolíticos.

${ }^{17}$ Gill, Gramsci, 1993, p. 22

${ }^{18}$ Un estudio excelente de los regímenes internacionales es Krasner, Third, 1985.

${ }^{19}$ Putnam, "Diplomacy", 1993, p. 436. 
El problema con este punto de vista es que no reconoce por necesidad los intereses estructurales en competencia dentro de un Estado, los niveles jerárquicos de los Estados actores (nacional, regional, local) y los diferentes ministerios (Hacienda contra Guerra, contra Relaciones Exteriores), los cuales con frecuencia no trabajan en forma concertada. Tampoco concede suficiente importancia a una más amplia definición del Estado que incluya los instrumentos de legitimidad-escuelas, iglesias, organizaciones civiles-y los instrumentos de represión. En otras palabras, las negociaciones en la vida real son mucho más complicadas que en un juego en dos niveles.

Aun si aceptamos que el juego en dos niveles se hace en lo fundamental sobre la soberanía y el poder del Estado, tenemos que reconocer que la soberanía es un concepto complicado. Stephen Krasner mostró hace poco que hay al menos cuatro diferentes tipos de soberanía: ${ }^{20}$

1. La soberania de interdependencia se refiere a la habilidad de un gobierno para realmente controlar las actividades dentro y a través de sus fronteras (incluyendo el movimiento de bienes, capital, ideas y vectores de enfermedad).

2. La soberanía interna se refiere a la organización de la autoridad dentro de una organización política dada.

3. La soberanía westfaliana se refiere a la exclusión de la autoridad externa; el derecho de un gobierno a

${ }^{20}$ Krasner, "Globalization", 1999. ser independiente de las estructuras de autoridad externas.

4. La soberanía internacional legal se refiere al reconocimiento de un Estado por otro; algunas entidades han sido reconocidas por otros Estados; otras no lo han sido. El reconocimiento se ha asociado a la inmunidad diplomática y al derecho de firmar tratados y unirse a organizaciones internacionales.

Según Krasner, estos cuatro acuerdos o definiciones de soberanía son precisos tanto en la lógica como en la práctica. Es posible tener uno sin los otros. Un Estado, Taiwán por ejemplo, puede tener soberanía westfaliana pero no poder gozar de soberanía internacional legal. Un Estado puede ser reconocido por otros Estados, pero carecer de soberanía interior y de interde. pendencia, así como de habilidad para controlar las actividades dentro y a lo largo de sus fronteras, situación que caracterizó a la mayor parte de América Latina en el siglo XIX y una parte de la cual todavía persiste (se piensa en Colombia). Un Estado puede gozar de soberanía internacional legal, puede ser reconocido por otros, pero no tener soberanía westfaliana.

Sin importar la forma en que se entienda la soberanía, es difícil construir una tesis sobre si las tendencias contemporáneas, en especial la globalización, están transformando la naturaleza del sistema. Nunca ha habido un pasado mítico en el que los Estados estuvieran seguros en el ejercicio ya sea de su control o de su autoridad. En lo particular los Estados más débiles han tenido siempre que luchar no sólo para mantener un dominio efectivo dentro y sobre sus fronteras, sino tam- 
bién para excluir la autoridad externa. La globalización ha despertado problemas nuevos y originales para la soberanía entendida como control, pero algunos Estados han enfrentado retos semejantes en el pasado. Algunos aspectos de la globalización, en especial la dispersión de ideas como los derechos de la mujer y la democracia, han influenciado la legitimidad de las formas institucionales nacionales particulares (un aspecto de la soberanía interna), pero ha habido retos similares en el pasado, incluyendo los derechos de las minorías y la autodeterminación nacional tras la primera guerra mundial. La amenaza al control estatal (soberanía de interdependencia), que algunos ven planteada por los niveles más altos de las transacciones internacionales, ignora el hecho de que los mercados de comercio y de capital estuvieron altamente integrados en el periodo anterior a la primera guerra mundial, y que la migración de trabajadores era todavía más pronunciada.

Así como por largo tiempo ha existido un problema de definición para entender la soberanía, así también ha existido un problema para entender al "Estado". Las preocupaciones de los Estados actores están empotradas en tradiciones, intereses de clase y discursos ideológicos. Es raro que las posturas realista y neorrealista en las relaciones internacionales miren dentro de la "caja negra" de la política interior, y si lo hacen, perciban un Estado monolítico racional que defiende los mal definidos "intereses nacionales", que poco tienen que ver con la realidad histórica. Las relaciones internacionales no están determinadas simplemente por elec- ciones racionales. ${ }^{21}$ En realidad, la irracionalidad, o al menos una racionalidad difuminada a través del prisma de intereses internos y percepciones cacofónicas, es más bien la regla.

La postura dependentista ha sido cada vez más atacada en los años recientes al exponerse la complejidad y las contradicciones de los regímenes de exportación. El liberalismo es visto como adaptable y razonable en tanto que muchos de los participantes en los regímenes de exportación se esforzaron por, y ocasionalmente lograron, un desarrollo nacional. ${ }^{22}$ Los liberales no eran comerciantes libres y partidarios automáticos del laissez faire, que hablaban con acento británico y acumulaban su dinero en París. Se plantearon debates muy serios e intensos retos al modelo de exportación. Sus resoluciones no fueron obvias ni predestinadas. Los debates que hicieron gran escándalo en México en los años 1890 sobre la alianza con Estados Unidos demuestran la amplia diversidad de intereses y perspectivas que estaban presentes en muchas regiones de América Latina. ${ }^{23}$

Una de las ventajas del enfoque de la dependencia, que tiende a caricaturizar las relaciones internacionales, es irónicamente que, si bien privilegió los papeles de las naciones en el sistema internacional, también reconoció

${ }^{21}$ Lake, Power, 1988, p. 87 y passim.

${ }^{22}$ Buenos ejemplos de esta tendencia se pueden encontrar en Love y Jacobsen, Guid. ing, 1988; Gootenberg, Between, 1989, y en Imagining, 1993. Topik y Wells, Second, 1998.

${ }^{23}$ Riguzzi, "Diplomacia", publicado en este mismo número. Véase también Topik, "México", en prensa. 
el nivel de interpenetración de los países y el punto hasta donde la nacionalidad era un concepto incómodo. Un concepto como "comprador", o el "lumpenbourgeoisie" de Frank, reconoce que un ciudadano de un país puede servir a los intereses de otra nación. ${ }^{24}$ Pero aun ello es fundamentalmente problemático porque las fidelidades del individuo no son por necesidad hacia un Estado-nación. En muchos lugares del continente americano, la familia y el pueblo van primero; la nación es un concepto distante. A veces, las identidades étnicas y de género son tan fuertes o más fuertes que las nacionales. Esto es cierto en la actualidad en el creciente movimiento indígena panamericano que comprende navajos, maya y mapuches, pero los límites nacionales entre los apaches de Estados Unidos y de México, o entre los mayas de Chiapas y Guatemala o Belice y Yucatán, nunca han sido reconocidos por sus habitantes. Las acciones fueron frecuentemente intraétnicas más que internacionales.

A veces la identidad nacional pertenece a una nación hace tiempo desaparecida. En Estados Unidos, los descendientes de los inmigrantes irlandeses seguían odiando a los británicos, demostrando este prejuicio en los lugares de votación y en las calles.

En México, a los "barcelonettes" se les consideraba partidarios del gobierno francés. Pero en este caso los intereses en debate los hacían actuar también como mexicanos. Los cónsules franceses se quejaban con firmeza de los inmigrantes franceses en México

${ }^{24}$ Frank, Lumpenbourgeoisie, 1974. provenientes de la ciudad de Barcelonette. Se les veía como estrechamente egoístas y antipatrióticos hacia Francia. Los mercaderes alpinos parecían no guardar fidelidad más que a otros individuos procedentes del pueblo de Barcelonette; por otra parte, los hombres de negocios eran mexicanos, ya que había sido en México en donde habían hecho su fortuna. En las diversas empresas en las que poseían una participación importante, como el Banco Nacional y el Banco de Londres, los bar. celonettes se conformaban con ser socios silenciosos, permitiendo que los inmigrantes españoles o alemanes dirigieran las compañias. Los barcelonettes estaban principalmente interesados en obtener ganancias y en mantener buenas relaciones con el gobierno mexicano. Raramente se comunicaban con el gobierno francés ni solicitaban mucha ayuda. ${ }^{25}$ Como observó Joseph Signoret, uno de los más ricos inmigrantes franceses:

Todos los directivos de las grandes casas mercantiles no podemos hacer prosperar nuestros negocios sin el auxilio del gobierno mexicano. Aquí, donde el capricho sustituye a todas las reglas, sería imposible luchar contra una administración hostil. ¿Cómo es posible formular opiniones libres en asuntos que

${ }^{25}$ Ministerio de Hacienda a Hanotaux, París, 7 de agosto de 1896; Benoit a Hanotaux, México, 13 de junio de 1897, en Archives du Ministère des Affairs Étrangères, Quai d'Orsay, París (en adelante MAE), Correspondence Politique, Mexico-France, vol. 1; Delcassé a Blondel, París, 12 de mayo de 1905; Dumaine al $\mathrm{Mi}$ nisterio de Asuntos Extranjeros, México, $8 \mathrm{de}$ marzo de 1908, en MAE, Correspondence Politique, México-France, vol. 26. 
interesan al gobierno, cuando la menor crítica expone a uno a ruinosas vejaciones? ${ }^{26}$

Muchos otros hombres de negocios "mexicanos" mostraron fidelidades mixtas que dependían de las circunstancias. La familia Martínez del Río (mexicanos que adoptaron la ciudadanía inglesa para protegerse) viene de inmediato a la memoria, como también Enrique Creel, Tomás Braniff y Hugo Scherer. Weetman Pearson, lord Cowdray, aunque claramente británico, era conocido en Londres como el "Miembro para México", ya que representaba muy bien los intereses mexicanos. ${ }^{27}$

Por otra parte, otros actores transnacionales reflejaban identidades ambiguas. Cuando la casa financiera "estadunidense" Speyer emitió un préstamo al gobierno mexicano, los diplomáticos franceses asumieron que Speyer estaba aliado con el gobierno estadunidense. Este error surgió de la suposición de que Speyer tenía una identidad nacional fija y de que trabajaba como una extensión de la política exterior de Estados Unidos. El mismo James Speyer explicó al ministro alemán, Wangenheim, que ofreció esas ventajosas condiciones a causa de la competencia de la Banque de Paris. ${ }^{28}$ En vez de servir

${ }^{26}$ Dumaine a Pinchon, México, 28 de julio de 1908 en MAE, Correspondence Politique, México-France, vol. 26.

${ }^{27}$ Young, Member, 1966; Collado, Burgue sia, 1987; Connolly, "Pearson", 1997; Walker, Kinship, 1986; Tenenbaum, "Merchants", 1979, pp. 317-340; Meyer, Majestad, 1991.

${ }^{28}$ Wangenheim a von Bülow, México, 29 de octubre de 1904, en Deutches Zentralarchiv, Postdam, DDR (en adelante DZP), Reichsamt des Innern 4383. a la política exterior de Estados Unidos, la casa bancaria llevaba a cabo una estrategia más amplia para obtener dominio particular de una amplia parte de las finanzas de México. Fue una decisión de negocios privada, no una iniciativa diplomática estadunidense. ${ }^{29}$

Es verdad que es cuestionable hasta dónde se puede considerar a Speyer como una casa estadunidense. James Speyer era por completo un financiero internacional. Nacido en Nueva York en 1861, partió a los tres años hacia Frankfurt, Alemania, para ser educado y entrenado como banquero, volviendo a Estados Unidos hasta 24 años después, para dirigir la sucursal de Speyer \& Co. Siguió teniendo participación en la casa alemana de Lazard Speyer Ellisen. Speyer tenía también una sucursal británica. La casa comercial y bancaria alemana, la más rica en Frankfurt a fines del siglo XVIII, había llegado a Estados Unidos en 1845 e hizo su fortuna mediante el financiamiento de ferrocarriles, especialmente el Southern Pacific y el Central Pacific de Collis P. Huntington, y aprovechando la ventaja del acceso que sus sucursales alemana e inglesa tenían al capital europeo. Hacia 1900, James Speyer era el segundo financiero más rico de Nueva York, después de J. P. Morgan. ${ }^{30}$

${ }^{29}$ En 1932 James Speyer trató acerca de un préstamo ante el Senado de Estados Unidos: "si no hubiésemos pensado que podríamos obtener algo de dinero, es posible que no nos hubiese interesado". Felicitado por su franqueza por un senador, Speyer contestó: "somos hombres de negocios". Sale, 1932, pp. 615-616.

${ }^{30}$ Ibid., pp. 609-611; Carosso, Investment, 1970, pp. 11-26, informa que, antes del surgimiento de los Rothschild, entre 1770 y 1800 , 


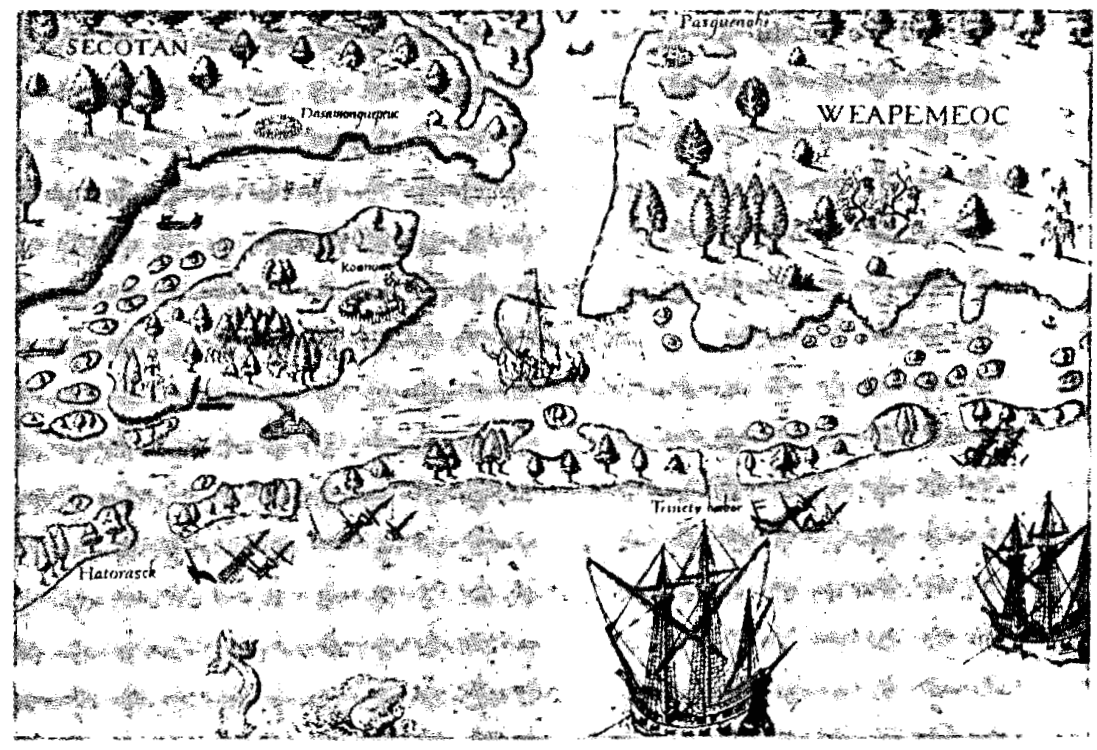

La casa Speyer se involucró con México como extensión a su actividad en los ferrocarriles. Las líneas mayores que Speyer ayudó a financiar en Estados Unidos empezaron a construir en México, durante los años 1880 , intentando monopolizar rutas cargueras fronterizas. $^{31}$ En 1901 Speyer pasó del financiamiento ferroviario a su posesión, cuando acudió en auxilio del Ferrocarril Nacional, en competencia con los intereses de Harriman-Rockefeller, contra quien Speyer había luchado en Es-

los Speyer eran los banqueros judios más ricos de Frankfurt. Hanrahan, Bad, 1985, vol. 1, pp. 113, 122, 123; Emden, Money, 1937, pp. 274277.

${ }^{31}$ Lamoreaux, Great, 1985. tados Unidos. Speyer financió la reorganización del Nacional y, con su compra de la Internacional, otra importante línea mexicana, a la larga llegó a dominar las líneas. Cuando Speyer intentó anexar la línea del Interoceánico, Limantour se opuso. Temiendo una monopolización estadunidenses de los principales ferrocarriles mexicanos, el ministro de Hacienda compró intereses para controlar las tres compañías. Prefirió aumentar la deuda del Estado antes que permitir que los consorcios yanquis dominaran el sistema nervioso ferroviario de México. Demostrando que temía más al capital industrial que al capital financiero, Limantour pidió prestado a la compañía a la que compraba los ferrocarriles para poder pagar las adqui- 
siciones. ${ }^{32}$ El préstamo fue un aperitivo para introducir a Speyer en el festín mexicano.

El ejemplo de los Speyer demuestra que las multinacionales no son nada nuevo. Esto era sobre todo cierto para los financieros que usualmente amalgamaban capital de numerosos países para financiar bonos o préstamos. ${ }^{33}$ Las compañías industriales hicieron lo mismo. Ceceña mostró su presencia de largo plazo en México. Pero el detalle es que ellos no eran sólo binacionales sino más bien internacionales. Para entender su manera de enfocar el mundo se tiene que reconocer que la lógica de sus imperios económicos no se detenía en la frontera; en la expresión de Mira Wilkins, se "desparramaba". ${ }^{34}$ Esto es más claro para los ferrocarriles estadunidenses y las compañías petroleras que entraron a México. Pero se debe tomar en cuenta una lógica corporativa global más amplia. Jonathan Brown demostró cómo las acciones de las compañías petroleras en México estaban relacionadas no sólo con las acciones del gobierno mexicano, sino también en competencia con la Royal Dutch Shell y los hallazgos petrolíferos en Venezuela. ${ }^{35} \mathrm{He}$ sugerido que a principios del siglo Xx México era un esce.

\footnotetext{
${ }^{32}$ Rakowitz a von Bülow, México 21 de diciembre de 1907 en DZP, Aus. Amt 1747; Díaz Dufoo, Limantour, 1910, p. 133; D'Olwer, "Vida", 1985, pp. 1068-1070; Hegeman, Mexico's, 1908 , p. 106; Parlee, "Porfirio", 1981, pp. 243246; Hanrahan, Bad, 1985, p. 123.

${ }^{33}$ Véase Marichal, Century, 1989, y Cameron, International, 1991.1

${ }^{34}$ Ceceña, México, 1970; Wilkins, Growth, 1991, y Wilkins y Schröter, Free-standing, 1998.

${ }^{35}$ Brown, Oil, 1993.
}

nario para compañías de Estados Unidos como las Guggenheim, Phelps-Dodge y Standard Oil, donde obtuvieron su primera experiencia latinoamericana, y de donde continuaron para establecer subsidiarias en toda América Latina. ${ }^{36}$ No es posible simplemente pensar en vías bipolares, sino más bien tomar en consideración ambos factores internos y el contexto global.

Hasta aquí he sugerido que los eventos y enfoques recientes han hecho problemáticos conceptos como Estado, nación y nacionalidad, que con frecuencia se pensaron estables y sin problema dentro de la historia diplomática.

Los grandes flujos de migración entre Centro América y México y entre México y Estados Unidos han causado problemas al concepto de límite fronterizo. A pesar de los esfuerzos estatales, no hay una línea clara entre los países. De hecho, cada vez más se ve el área fronteriza como una área separada casi liminar que no es enteramente parte de algún país. ${ }^{37}$ Esto, claro está, no es nada nuevo. De hecho, a las áreas fronterizas se les dieron exenciones legales especiales a finales del siglo XIX para alentar su desarrollo. Mineros, hacendados, bandidos e indios se movían con libertad hacia adelante y hacia atrás. En realidad, compañías como Phelps-Dodge tenían minas a ambos lados de la frontera y utilizaron la caballería del estado de Arizona para terminar una huelga en Cananea, México. Dorothy Kering ha hecho un estudio

\footnotetext{
${ }^{36}$ Topik, "Emergence", 1992. Véase también Weiner, "U. S."

${ }^{37}$ Beezley, Gran, 1994; Lorey, "U. S."
} 
imaginativo y detallado de las conexiones entre Estados Unidos y México, que se hallaban detrás de la Colorado River Land Company en Baja California, desarrolladas por la misma gente que había desarrollado el valle de San Fernando al norte de Los Ángeles. ${ }^{38}$

Y la población no estaba sólo bajo la influencia del gobierno nacional del país en el que se encontraba. Gil González ha estudiado a los cónsules mexicanos en California y los encontró muy activos organizando trabajadores mexicanos e intentando resolver huelgas. ${ }^{39}$ Dirk Raat mostró la importancia del sureste de Estados Unidos como un escenario de los "revoltosos" mexicanos que buscaban derrocar al gobierno de México, mientras que Colin McLachlan demostró que Ricardo Flores Magón, quien se sublevó para reformar México, se enredó en la política radical estadunidense cuando vivía en California. ${ }^{40}$

Los recientes trabajos de historia cultural han abierto también áreas nuevas para la investigación y métodos nuevos en las relaciones internacionales. El efecto de un país en otro ha sido mucho más que el solamente político o económico. Raquel Rubio Goldsmith traza la manera en que el papel central que el patio tiene en la cultura mexicana arraigó en el suroeste de Estados Unidos. ${ }^{41}$ Jeff Garcilazo examinó la migración de la cultura mexicana del nor1988.

${ }^{38}$ Kerig, "Yankee", 1998; Hall, Revolution,

${ }^{39}$ González, Mexican, 1999.

${ }^{40}$ Raat, Revoltosos, 1988, y Mexico, 1996; MacLachlan, Anarchism, 1991.

${ }^{41}$ Rubio, "Seasons", 1994, pp. 140-156. te, llevada por traqueros ${ }^{42}$ que trabajaban en los ferrocarriles de Estados Unidos. Miguel Tinker Salas hizo un estudio similar buscando también las repercusiones en México. ${ }^{43}$

La sexualidad es también uno de los valores importantes que cruzan la frontera. Vicky Ruiz, Douglas Montero y George Sánchez muestran la importancia de la percepción de los papeles del género, que fueron importados de México a California. ${ }^{44}$

Sin embargo, ya que no hay una sola cultura mexicana, en Estados Unidos existen versiones competitivas de un nuevo híbrido en formación. Michael Kearney ha mostrado cómo los trabajadores mixtecos de Oaxaca, en Baja y Alta California, desarrollan nuevas percepciones de identidad que han utilizado para afectar al sistema político al volver a Oaxaca. La tendencia reciente de los jóvenes inmigrantes salvadoreños en Los Ángeles, de crear pandillas callejeras como la Mara Salvatrucha que, a su vez, se vuelve políticamente importante al regresar a El Salvador, señala otro fenómeno fronterizo que merece una investigación histórica. ${ }^{45}$ Es claro que las identidades nacionales no son fijas y estables. Las relaciones internacionales las afectan al igual que ellas afectan a las relaciones internacionales.

\footnotetext{
${ }^{42}$ De track, vía férrea. El que trabaja en los ferrocarriles [ $\mathrm{N}$. del $\mathrm{T}$.]

43 Garcilazo, "Traqueros", 1995; Tinker, Shadow, 1997.

${ }_{44}$ Monroy, Rebirtb, 1999, y Tbrown, 1990; Sánchez, Becoming, 1993; Ruiz, Shadows, 1998

${ }^{45}$ Kearney, "Integration", 1986. Véase también su Reconceptualizing, 1996.
} 
Teresa Romero trabaja en un sugerente estudio que muestra los resultados involuntarios de las relaciones internacionales. Observando a los misioneros protestantes que van de Estados Unidos a México con la mira de inculcar la religión estadunidense y la perspectiva del dominio masculino, encontró que también procuraron educar a las jóvenes mexicanas como una forma para atraer a sus familias al redil. Para lograrlo, llevaron a mujeres estadunidenses solteras quienes, mediante la supuesta enseñanza de la sumisión, mostraron con su mera presencia un papel independiente para las mujeres. Numerosas mujeres educadas por las misioneras estadunidenses llegaron a ser líderes en el movimiento femenino mexicano. ${ }^{46}$

Este ejemplo señala también la importancia de las agencias no gubernamentales en las relaciones internacionales. Desde hace tiempo se ha reconocido a los inversionistas extranjeros, aun cuando por lo general se les ha relegado a la historia económica o empresarial, más que a las relaciones internacionales. No obstante, varias áreas, como la de la industria petrolera, han producido estudios complejos sobre la relación entre negocios y diplomacia. Los estudios de Jonathan Brown, Linda Hall, Lorenzo Meyer y Alan Knight vienen a la memoria. ${ }^{47}$ Estudios sobre Centro América como el de Thomas Schoonover, The banana men, han señalado esto. Estudios más generales sobre la diplomacia de los negocios, como el de

${ }^{46}$ Romero, "Religious".

${ }^{47}$ Meyer, Grupos, 1973, y México, 1981; Brown y Knigth, Mexican, 1992; Hall, Oil, 1995.
Thomas F. O'Brian, The revolutionary mission american enterprise in Latin America, 1900-1945, amplían también la red de las relaciones internacionales. ${ }^{48}$

A veces, las compañías diseñan nuevas formas de hacer negocios que tienen consecuencias importantes para el país anfitrión. Julio Moreno ha demostrado que Sears de México reconceptualizó la naturaleza de la venta al menudeo y utilizó la contradicción del nacionalismo mexicano y su deseo de modernidad en beneficio de la compañía. Sears simplemente no podía actuar en México como lo había hecho en Estados Unidos. Fue transformada. Moreno muestra también cómo J. Walter Thompson de México tuvo que cambiar sus tácticas publicitarias, empleando mayoritariamente a mexicanos, quienes tenían mayor familiaridad con la cultura local. ${ }^{49}$

La recepción de ideas y bienes se vuelve complicada cuando cruza las fronteras. The allure of the foreign, de Ben Orlove, muestra cómo la cultura de la mercancía entró a América Latina. El estudio de Rick Weiner acerca de las ideas económicas en el México porfiriano prueba que, lejos de ser servil imitadora del pensamiento europeo y estadunidense, la elite porfiriana estaba muy preocupada por el papel subordinado de México en la economía mundial y por la sombra de Estados Unidos. Más que aceptar la idea de un liberalismo de libre comercio,

\footnotetext{
${ }^{48}$ Schoonover y Langley, Banana, 1995; O'Brian, Revolutionary, 1996; Kinzer, Bitter, 1982. Véase también Buchenau, Shadow, 1996.

${ }^{49}$ Moreno, "Search"y "Marketing", 1998.
} 
buscaba un desarrollo guiado por el Estado. ${ }^{50}$

Podría ir mucho más lejos ennumerando trabajos interesantes y provocativos que abren nuevas formas para considerar las relaciones internacionales. Concluiré con una antología reciente que combina muchos de los nuevos enfoques, Close encounters of empire. Writing the cultural bistory of U.S.-latin american relations, compilada por Gilbert Joseph, Catherine LeGrand y Ricardo Salvatore, ${ }^{51}$ volumen inspirado en la observación de Emily Rosenberg de que la historia diplomática debe ser historia internacional y enfocarse a los asuntos culturales. ${ }^{52}$ Esta colección reúne el interés posmoderno en la descentralización y las representaciones con formas nuevas de considerar las relaciones internacionales, no como extensiones de modelos previos. Sus estimulantes artículos subrayan el hecho de que muchas de las fluidas fronteras, las formas de Estados en competencia y las múltiples personalidades e identidades que hoy encontramos, han existido desde bastante tiempo atrás. También muestra que las relaciones internacionales incluyen mucho más que sólo a los diplomáticos, y que aun los diplomáticos son impulsados por cuestiones de género, identidad y cultura, así como por consideraciones políticas reales.

Ello no significa que esté argumentando a favor de una consideración poscolonial o posmoderna de las relacio-

\footnotetext{
${ }^{50}$ Weiner, "Competing", 1999; Orlove, Allure, 1997.

51 Joseph, LeGrand y Salvatore, Close, 1998.

52 Rosenberg, "Walking", 1990.
}

nes internacionales, con la cultura suplantando la economía política. Eso, como hace notar William Roseberry, sería un error. La tarea es "construir y extenderse en tempranos entendimientos y formas de análisis por medio del diálogo y el argumento, más que suplantarlos o desplazarlos simplemente", ${ }^{5.3}$

Tomando en cuenta los retos del mundo moderno, valiéndose de la crisis de la epistemología para cuestionar viejas verdades y acomodar argumentos con nuevos componentes, tenemos la oportunidad de rejuvenecer la historia diplomática. Las relaciones internacionales pueden -y deben- ser uno de los campos más atractivos de la investigación.

\section{ARCHIVOS}

MAE Archives du Ministère des Affaires Étrangères, Quai d'Orsay, París.

DZP Deutsches Zentralarchiv, Potsdam.

\section{BIBLIOGRAFÍA}

-Bandeira, Moniz, Presenca dos Estados Unidos no Brasil (Dois séculos de bístoria), Civilizaçao Brasileira, Río, 1967.

-Becker, William, The dynamics of business-government relations: industry and exports; 1893-1921, University of Chicago Press, Chicago, 1982.

-Beezley, William y Colin MacLachlan, El gran pueblo: a bistory of greater Mexico, Prentice-Hall, Englewood Cliffs, Nueva Jersey, 1994,

-Beisner, Robert, "Comment", American Historical Review, vol. 83, núm. 3, 1978, pp. 674-679.

$\$ 3$ Roseberry, “Social”, 1998, p. 516. 
-Bemis, Samuel Flagg, A diplomatic bistory of the United States, H. Holt and Co., Nueva York, 1936.

-Benjamin, Jules R., "The framework of $U$. S. relations with Latin America in the twentieth century: an interpretative essay", Diplomatic History, vol. 11, núm. 2, 1987, pp. 91-112.

-Brown, Jonathan y Alan Knight (comps.), The mexican petroleum industry in the twentieth century, University of Texas Press, Austin, 1992.

-Brown, Jonathan, Oil and revolution in Mexico, University of California Press, Berkeley, 1993.

-Buchenau, Jurgen, In the shadow of the giant: the making of Mexico's central american policy, 1898-1930, University of Alabama Press, Tuscaloosa, 1996.

-Cameron, Rondo y V. I. Bovykin, International banking 1870-1914, Oxford University Press, Nueva York, 1991.

-Campbell, Charles S., The transformation of american foreign relations, 18651900, Harper and Row, Nueva York, 1976.

-Carosso, Vincent P., Investment banking in America; a bistory, Harvard University Press, Cambridge, 1970.

-Ceceña, José Luis, México en la órbita imperial, El Caballito, México, 1970.

-Collado, María del Carmen, La burguesia mexicana, el emporio Braniffy su participación política, Siglo Veintiuno, México, 1987.

-Connolly, Priscilla, "S: Pearson \& Son: contratista de obras públicas" en Carlos Marichal y Mario Cerutti (comps.), Historia de las grandes empresas en México, 1850-1930, Fondo de Cultura Económica, México, 1997.

-D'Olwer, Luis Nicolau et al., La vida económica. Historia moderna de México. El porfiriato, Hermes, México, 1985.

-Díaz Dufoo, Carlos, Limantour, E. Gómez de la Puente, México, 1910.

-Emden, Paul H., Money powers of Europe in the nineteenth and twentietb centuries, S. Low, Marston \& Co., 1937.
-Field, James A., Jr., "American imperialism: whe 'worst chapter' in almost any book", American Historical Review, vol. 83, núm. 3, 1978, pp. 644-669.

-Frank, André Gunder, Lumpenbourgeoisie, lumpendevelopment, Monthly Review Press, Nueva York, 1974.

-Frank, Thomas, "It's globalicious! Two servings, half-baked, of the new economy", Harper's Magazine, 1999 , pp. 72-75.

-Friedman, Thomas, The lexus and the olive tree: understanding globalization, Farrar, Straus and Giroux, Nueva York, 1998.

-Garcilazo, Jeffrey, "Traqueros: mexican railroad workers in the United States, 18701930", tesis doctoral, Universidad de California, Santa Bárbara, 1995.

-Gardner, Lloyd C., Walter LaFeber y Thomas McCormick, Creation of the american empire, Rand, McNally and $\mathrm{Co}$., Chicago, 1973.

-Gill, Stephen (comp.), Gramsci. Historical materialism and international relations, Cambridge University Press, Londres, 1993.

-González, Gilbert G., Mexican consuls and labor organizing: imperial politics in the american southwest, University of Texas Press, Austin, 1999.

-Gootenberg, Paul, Between silver and guano: commercial policy and the State in postindependence Peru, Princeton University Press, Princeton, 1989.

Imagining development; economic ideas in Peru's "fictitious prosperity" of guano, 1840-1880, University of California Press, Berkeley, 1993.

-Graebner, Norman, An uncertain tradition: american secretaries of State in the twentieth century, McGraw-Hill, Nueva York, 1961.

-Hall, Linda y Donald Coerver, Revolution on the border: the United States and Mexico 1910-1920, University of New Mexico Press, Albuquerque, 1988.

Hall, Linda, Oil, banks, and politics: the United States and postrevolutionary 


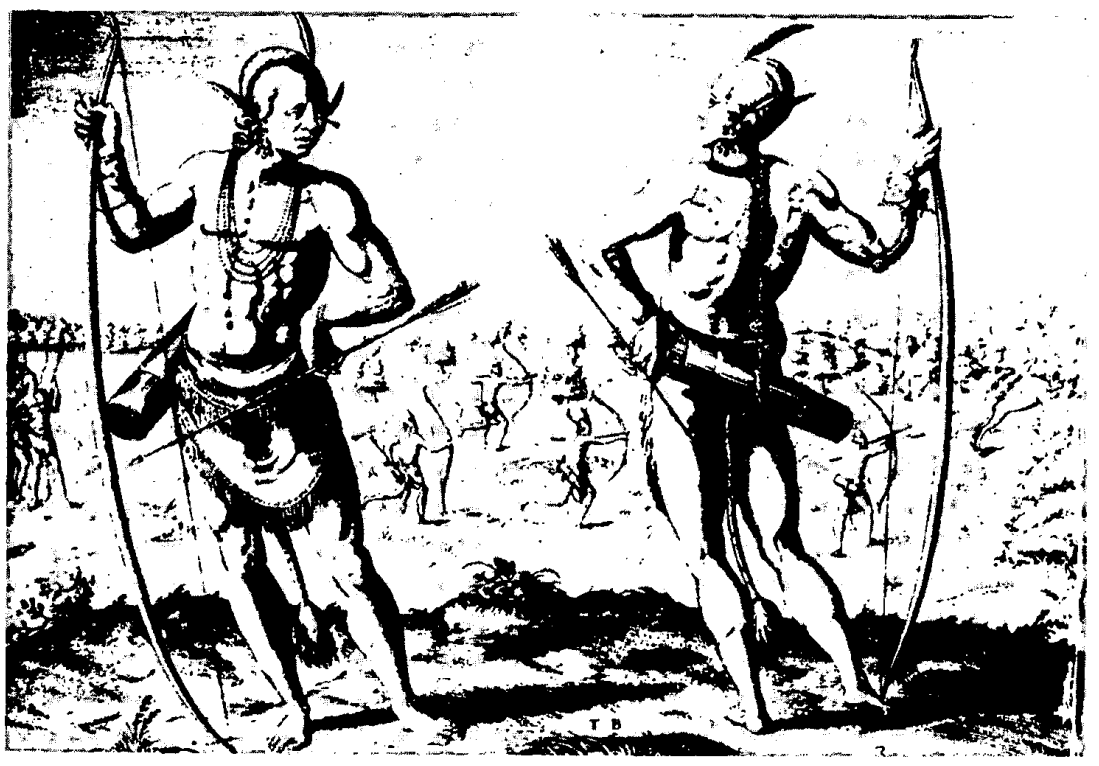

Mexico 1917-1924, University of Texas Press, Austin, 1995.

-Hanrahan, Gene Z., The bad yankee; el peligro yankee. American entreprenuers and financiers in Mexico, Documentary Publication, Chapel Hill, Carolina del Norte, 1985.

-Hegeman, Werner, Mexico's Ubergang zur Gold Wabrung, J. Cottsche Buchhandlung, Stuttgart y Berlín, 1908.

-Holbo, Paul S., "Economics, emotion, and expansion: an emerging foreign policy" en H. Wayne Morgan (comp.), The Gilded Age, Syracuse University Press, Syracuse, 1971, pp. 199-221.

-Jones, Stanley L., The presidential election of 1896, University of Wisconsin Press, Madison, 1964.

-Joseph, Gilbert M., Catherine C. LeGrand y Ricardo D. Salvatore (comps.), Close encounters of empire. Writing the cultural bistory of U. S. -latin american relations, Duke University Press, Durham, Carolina del Norte, 1998.

-Kearney, Michael, "Integration of the Mixteca and the western U. S.-mexican border region via migratory wage labor" en Ina Rosenthal Urey (comp.), Impacts of U. S.-mexican relations, University of California, San Diego, 1986.

- Reconceptualizing the peasantry: antbropology in global perspective, Westview, Boulder, Colorado, 1996.

-Kerig, Dorothy, "Yankee enclave: the Colorado River Land Company and mexican agrarian reform, 1902-1944", tesis doctoral, Universidad de California, Irvine, 1988.

-Kiernan, V. G., Marxism and imperialism, Edward Arnold, Londres, 1974.

-Kinzer, Stephen, Bitter fruit, the untold story of the american coup in Gua- 
temala, Doubleday, Garden City, Nueva Jersey, 1982.

-Krasner, Stephen, Defending the national interest: raw materials investment and U.S. foreign policy, Princeton University Press, Princeton, 1978.

, "Globalization and sovereignty" en David A. Smith, Dorothy J. Solinger y Steven C. Topik, States and sovereignty in the global economy, Routledge, Londres, 1999.

$$
\begin{aligned}
& \text {, The third world against glo- } \\
& \text { bal liberalism: a structural conflict, Uni- } \\
& \text { versity of California Press, Berkeley, } 1985 \text {. } \\
& \text {-LaFeber, Walter, "Comment", Ameri- } \\
& \text { can Historical Review, vol. 83, núm. } 3 \text {, } \\
& \text { 1978, pp. } 669-674 .
\end{aligned}
$$

, The Cambridge bistory of american foreign relations. Volume II. The american search for opportunity, 1865 1913, Cambridge University Press, Nueva York, 1993.

The new empire. An interpretation of american expansion, 18601898, Cornell University Press, Ithaca, Nueva York, 1963.

-Lake, David A., Power, protection and free trade; international sources of $U$. S. commercial strategy, 1887-1939, Cornell University Press, Nueva York, 1988.

-Lamoreaux, Naomi, The great merger movement in american business, 1895. 1904, Cambridge University Press, Cambridge, Inglaterra, 1985.

-Lewis, William Roger (comp.), Imperialism: the Robinson and Gallegber controversy, New Viewpoints, Nueva York, 1976.

-Lorey, David E., "The U. S.-mexican border in the twentieth century", manuscrito.

-Love, Joseph y Nils Jacobsen (comp.), Guiding the invisible band: economic liberalism and the State in latin american bistory, Praeger, Nueva York, 1988.

-Maclachlan, Colin, Anarchism and the mexican revolution: the political trials of Ricardo Flores Magon in the United Sta. tes, University of California Press, Berkeley, 1991.

-Marichal, Carlos, A century of debt crisis in Latin America. From independence to the Great Depression, 1820-1930, Princeton University Press, Princeton, Nueva Jersey, 1989.

-Markusen, Ann, Regions: the economics and politics of territory, Rowman and Littlefield, Totowa, Nueva Jersey, 1987.

-May, Ernest, American imperialism: a speculative essay, Atheneum, Nucva York, 1968.

-McCormick, Thomas J., Cbina market: America's quest for informal empire, 18931901, Quadrangle Books, Chicago, 1967.

-Meyer, Lorenzo, Los grupos de presión extranjeros en el México revolucionario, 1910-1940, Secretaría de Relaciones Exteriores, México, 1973.

, México y los Estados Unidos en el conflicto petrolero, 1917-1942, El Colegio de México, México, 1981.

- Su majestad británica contra la revolución mexicana, 1900-1950: el fin de un imperio informal, El Colegio de México, México, 1991.

-Monroy, Douglas, Rebirtb: mexican Los Angeles from the great migration to the great depression, University of California Press, Berkeley, 1999.

University of California, Berkeley, 1990.

-Moreno, Julio, "In search of markets, diplomacy, and middle class consumers: Sears Roebuck in Mexico city and the forging of commercial relations between Mexico and the U. S. in the post world war era", manuscrito.

- "Marketing in Mexico: Sears Roebuck Company, J. Walter Thompson and the culture of north american commerce in Mexico during the 1940's", tesis doctoral, Universidad de California, Irvine, 1998.

-Nelson, Keith, "The 'warfare State'. history of a concept", Pacific Historical Re- 
view, vol. 40, núm. 2, mayo de 1971, pp. 127-143.

-O'Brian, Thomas F., The revolutionary mission: american enterprise in Latin America, 1900-1945, Cambridge, Nueva York, 1996.

-Orlove, Benjamin, The allure of the foreign: imported goods in post colonial America, University of Michigan Press, Ann Arbor, 1997.

-Parlee, Lorena, "Porfirio Diaz, railroads and development in northern Mexico: a study of government policy toward the Central and National railroads, 1876-1910", tesis doctoral, Universidad de California, San Diego, 1981.

-Putnam, Robert D., "Diplomacy and domestic politics: the logic of two-level games" en Peter B. Evans, Harold K. Jacobsen y Robert D. Putnam (comps.), Doubleedged diplomacy: international bargaining and domestic politics, University of California Press, Berkeley, 1993.

-Raat, Dirk W., Los revoltosos: rebeldes mexicanos en los Estados Unidos 19031923, Fondo Cultura Económica, México, 1988.

- Mexico and the United States. Ambivalent vistas, University of Georgia Press, Athens, 1996.

-Romero, María Teresa, "Religious education and cultural conversion: american protestant schools in Mexico, 1880-1930", tesis doctoral en curso, Universidad de California, Irvine.

-Roseberry, William, "Social fields and cultural encounters" en Gilbert M. Joseph, Catherine C. LeGrand y Ricardo D. Salvatore (comps.), Close encounters of empire. Writing the cultural bistory of $U$. S. . latin american relations, Duke University Press, Durham, Carolina del Norte, 1998, pp. 515-524.

-Rosenberg, Emily, Spreading the american dream: american economic and cultural expansion, 1890-1945, Hill and Wang, Nueva York, 1982.
"Walking the borders", $D i$ -

plomatic History vol. 14, núm. 4, 1990, pp. 565-573.

-Rubio Goldsmith, Raquel, "Seasons, seeds, and souls: mexican women gardening in the american Mesilla, 1900-1940" en Heather Fowler-Salamini y Mary Kay Vaughn (comps.), Women of the mexican countryside, University of Arizona Press, Tucson, 1994, pp. 140-156.

-Ruiz, Vicki, From out of the shadows: mexican women in twentietb century America, Oxford University Press, Nueva York, 1998.

-Sale of foreign bonds or securities in the United States: hearings before the Committee on Finance, United States Senate, 72 Congreso, 1a. sesión, parte 2, enero 4.7 de 1932, Government Printing Office, Washington, 1932.

-Sanchez, George, Becoming mexican american: ethnicity, culture and identity in chicano Los Angeles, 1900-1945, Oxford University Press, Nueva York, 1993.

-Schoonover, Thomas y Lester D. Langley, The banana men. American mercenaries and entrepreneurs in Central America, 1880-1930, University of Kentucky, Lexington, 1995.

-Sklar, Martin, The corporate reconstruction of american capitalism, 1890-1916; the market, the law and politics, Cambridge University Press, Nueva York, 1988.

-Tenenbaum, Barbara, "Merchants, money and mischief: the british in Mexico, 1821-1862", The Americas, vol. 35, núm. 3, 1979, pp. 317-340.

-Thurow, Lester, Building wealth: the wew rules for individuals, companies, and nations in a knowledge-based economy, Harper Collins, Nueva York, 1999.

-Tinker Salas, Miguel, In the shadow of the eagles: Sonora and the transformation of the border during the porfiriato, University of California Press, 1997.

-Topik, Steven C., "The emergence of finance capital in Mexico" en Virginia Gue- 
dea y Jaime E. Rodríguez O. (comps.), Cinco siglos de bistoria de México, Instituto Mora, México, 1992.

y Allen Wells, The second conquest of Latin America; coffee, benequen, and oil during the export boom 1850-1930, University of Texas Press, Austin, 1998.

Trade and gunboats. The United States and Brazil in the age of empire, Stanford University Press, Stanford, 1996.

"When Mexico had the blues: a transatlantic tale of bonds, bankers, and nationalists", American Historical Review (en prensa).

-Walker, David, Kinship, business and politics. The Martinez del Rio family in Mexico, 1823-1867, University of Texas Press, Austin, 1986.

-Weiner, Richard, "Competing market discourses in porfirian Mexico", Latin American Perspectives, vol. 26, núm. 1, 1999, pp. 22-43.
"U. S. businesses turn their eyes to Mexico in the early twentieth century", manuscrito.

-Wilkins, Mira (comp.), The growth of multinationals, E. Elgar, Aldershot, Hants, Inglaterra y Brookfield, Vermont, Estados Unidos, 1991.

The free-standing company in the world economy, 1830-1996, Oxford University Press, Oxford, Nueva York, 1998.

- The bistory of foreign investment in the United States to 1914, Harvard University Press, Cambridge, Massachusetts, 1989.

-Williams, William A., The tragedy of american diplomacy, World Publishing Co., Cleveland, 1959.

-Young, Desmond, Member for Mexico, a biography of Weetman Person, Cassell, Londres, 1966. 